\title{
Un itinerario por los proyectos editoriales del anarquismo en Argentina: cambios, maniobras y permanencias
}

\author{
An itinerary throughout Anarchism's editorial projects in Argentina: changes, \\ maneuvers and continuities
}

\author{
Lucas Domínguez Rubio*
}

\begin{abstract}
Resumen: Si en los momentos de mayor efervescencia política aumentan las ediciones de folletos y periódicos, a su vez pueden observarse ciertas diferencias en la vida de un movimiento político según el tipo de intervención que proponen sus publicaciones. El auge de la actividad editora del movimiento anarquista en Buenos Aires precedió a su indudable importancia gremial durante los primeros años del siglo XX. Mientras hasta ese momento las pocas "ediciones populares" apuntaban a publicar obras literarias, el anarquismo generó un novedoso circuito de textos que planteaban una relación sin precedentes entre imprentas, política, autores, editores y lectores. De manera que sus prácticas descentralizadas de financiación y difusión dieron a conocer un amplio abanico temático de preocupaciones políticas que con el correr de las décadas resultarían constitutivas de los intereses editoriales del movimiento libertario: gremialismo, sexualidad, emancipación de la mujer, naturismo, educación, antinacionalismo, antimilitarismo y anticlericalismo. Este trabajo propone un itinerario por los principales emprendimientos editoriales libertarios - La questione sociale, Biblioteca Sociológica, La Protesta, Fueyo, Ángel Zucarrelli, Argonauta, Atlas, La Palestra, Nervio, Imán, Tupac, Reconstruir, Américalee y Proyección- con el fin de determinar las especificidades propias de estas editoriales dentro de la historia del libro en Argentina.
\end{abstract}

Palabras clave: Historia del libro y la edición, Izquierda argentina, Anarquismo argentino, editores

\begin{abstract}
In times of political effervescence, the number of pamphlets and newspapers editions tends to increase. Accordingly, the different instances in the life of a political movement can be traced through the type of intervention proposed by its publications. The rise of the anarchist movement publishing activity in Buenos Aires preceded its relevance within trade union during the first years of the 20th century. Until that moment the few "popular editions" published in the country consisted in literary works. In that context, Anarchism generated a novel circuit of texts through which an unprecedented relationship between printers, politics, authors, publishers and readers was established. Therefore, the decentralized financing and dissemination practices of the anarchist publishing movement revealed a broad thematic range of political concerns, that would finally shape, over the decades, the editorial interests of
\end{abstract}

\footnotetext{
*Argentino, CeDInCI/UNSAM - CONICET. lucaslmdr@autistici.org
} 
the libertarian movement: gremialism, sexuality, emancipation of women, naturism, education, anti-nationalism, anti-militarism and anticlericalism. This work provides an itinerary for the main libertarian publishing projects - La questione sociale, Sociological Library, La Protesta, Fueyo, Ángel Zucarrelli, Argonauta, Atlas, La Palestra, Nero, Imán, Tupac, Rebuild, Américalee and Projection- in order to determine the specific characteristics of these publishers within the history of the book and textual editing in Argentina.

Keywords: history of the book and textual editing, Argentine left thought, Argentine anarchism, Publishers

Recibido: 3 enero 2017

Aceptado: 8 marzo 2017

\section{Introducción: una cultura editorial libertaria}

Entre 1895 y 1901 el auge de la actividad editora del movimiento anarquista en Buenos Aires precedió a su indudable importancia gremial durante los primeros años del siglo XX. Mientras hasta ese momento las pocas "colecciones populares" existentes editaban únicamente obras literarias, el anarquismo generó un novedoso circuito de textos que planteaban una relación sin precedentes entre imprentas, política, autores, editores y lectores. En años de una fuerte llegada de inmigrantes que coincidió con el momento de ampliación del público lector en Argentina, ávidos grupos editores pusieron en diálogo a nuevos autores extranjeros en publicaciones de amplio alcance. Sus prácticas descentralizadas de financiación y difusión dieron a conocer un abanico temático de preocupaciones políticas que con el correr de las décadas resultarían constitutivas de los intereses editoriales libertarios: lucha obrera, sexualidad, emancipación de la mujer, educación, naturismo, antipatriotismo, antimilitarismo y anticlericalismo. De allí en más, la actividad editorial ya no resultaría exclusiva de los círculos cercanos a los funcionarios estatales y otros miembros de la elite.

$\mathrm{Si}$ en los momentos de mayor efervescencia política aumentan las ediciones de folletos y periódicos, a su vez pueden observarse ciertas diferencias en la vida de un movimiento político según el tipo de intervención que proponen sus publicaciones. Si bien la cantidad de folletos y libros editados por el movimiento anarquista local fue en aumento entre 1895 y 1901, en los años posteriores la gran mayoría de los grupos libertarios priorizó la edición de publicaciones periódicas gremiales y literarias. Así, después del 900, aparecieron ediciones libertarias de poesía y teatro, y aún más adelante, después del '20, también cuentos y relatos a través de los folletines seriados. Tan sólo en los años finales de la década del treinta algunas editoriales libertarias publicaron novelas. A partir de este momento, la propuesta coyuntural del semanario anarquista se reemplazó por la edición de 
series monográficas y el campo cultural libertario fue en gran medida dominado por proyectos editoriales que apuntaron al formato libro. En definitiva, con el correr de las décadas la cantidad y tirada de los periódicos anarquistas menguaron considerablemente, mientras aumentó el tamaño y la calidad de los libros editados.

Este texto propone un itinerario cronológico por el desarrollo editorial del movimiento anarquista argentino en busca de identificar sus características propias y sus modificaciones. Con este objetivo, un recorrido por los principales emprendimientos editoriales libertarios —La questione sociale, Biblioteca Sociológica, La Protesta, Fueyo, Ángel Zucarrelli, Argonauta, La Palestra, Nervio, Imán, Tupac, Reconstruir, Américalee y Proyección- permitirá comenzar a pensar las especificidades de estas propuestas editoriales dentro de la historia del libro en Argentina. De esta manera, metodológicamente el presente trabajo parte de las preguntas historiográficas de la historia del libro y la edición. Lejos de ser considerado solamente un nuevo enfoque posible sobre el tema, estas páginas apuntan a resaltar que las prácticas editoriales del movimiento libertario no sólo revistieron ciertas particularidades idiosincráticas, sino que también sirven para explicar el gran alcance inicial que tuvieron sus impresos en forma de folletos financiados por suscripción y repartidos gratuitamente. Esta táctica se modificó parcialmente después de 1920. A partir de allí, por un lado, durante algunos años algunos emprendimientos libertarios apuntaron a instalar autores anarquistas mediante folletos seriados de amplia difusión. Mientras, por otro lado, una seguidilla de proyectos editoriales comenzaron a publicar libros de gran formato que consolidarían una cultura libertaria en las décadas posteriores ${ }^{1}$.

\section{Difusión de los primeros textos libertarios}

Los primeros contactos con seguidores de Bakunin en Argentina deben rastrearse a fines del año 1870 en la correspondencia que mantienen algunos miembros de la Sociedad Tipográfica Bonaerense con el Consejo Federal de la Región Española vinculada a la Asociación Internacional de Trabajadores (AIT), aunque estos intercambios no hayan pasado del envío de algunas publicaciones. En conjunción con la correspondencia de Wilmart, por lo general se toman las cartas enviadas por el propagandista de la sección

\footnotetext{
${ }^{1}$ Una versión muy preliminar de este trabajo ha sido publicada en las Actas del Primer Coloquio sobre el Libro y la Edición realizado en La Plata en el 2012. Entre otros trabajos que se citan en la bibliografía final, para la historia editorial argentina principalmente he utilizado: De Zagastizábal, Leandro, La edición de libros en la Argentina, Eudeba, 1995; y los estudios compilados en De Diego, José (dir.), Editores y políticas editoriales en Argentina: 1880-2000. Buenos Aires: FCE, 2006. Sobre editoriales anarquistas argentinas me apoyo en los trabajos ya realizados: Pérez, Pablo - Villasenin, Hernán - Jofre, Liliana, "Las armas y las letras. Un recorrido por las ediciones anarquistas", La Biblioteca 2, 2006; de la Rosa, Fernanda, "Entre la militancia y los proyectos editoriales. La labor de Diego Abad de Santillán dentro del anarquismo argentino, 19201930", IV Jornadas de Historia de las Izquierdas, Buenos Aires, CeDInCI, 2007; Graciano, Osvaldo, "La escritura de la realidad. Un análisis de la tarea editorial y del trabajo intelectual del Anarquismo argentino, entre los años 30 y el Peronismo", Izquierdas 12, 2012; y Anapios, Luciana, "Prensa y estrategias editoriales del movimiento anarquista en la Argentina de entreguerras", Anuario del Instituto de Historia Argentina, vol. 16, n $2,2016$.
} 
uruguaya -H. A. Juanes - y por F. C. Galcerán para afirmar que la seccional porteña fue "marxista", y sólo entre los panaderos Juanes había "encontrado una atmósfera favorable a las sociedades de socorros y resistencia"2. De manera que en Argentina hacia 1872 habría interesados en los textos de Proudhon y Bakunin, pero estos no habrían estado mayormente organizados.

Como lo hicieron sin muchas más opciones Nettlau y Santillán, y de ahí en más una larga lista de investigadores, básicamente hay que confiar en el mismo texto de José Ingenieros del Almanaque socialista de La Vanguardia de 1899 para sostener que en 1876 se fundó en Buenos Aires el Centro de Propaganda Obrera. Según Ingenieros, este centro fue bakunista y en 1879 habría publicado el primer folleto libertario local titulado Una idea, en el que se daba cuenta de los principios generales de la Internacional y el Pacto de Jura, por lo que habría incluido las críticas de Bakunin al marxismo ${ }^{3}$.

Aparecido en el mismo año que el folleto Una idea y el igualmente inhallable periódico La Vanguardia, habitualmente se ha considerado a El descamisado: periódico rojo (Buenos Aires, 1879) el primer medio ácrata que se conserva. Del año siguiente en algunos viejos catálogos aparece nombrado un periódico llamado La anarquía que aparentemente se publicó en 1880 en Buenos Aires pero sobre el cual carecemos de más datos. Y, si bien desde esa fecha se produce un importante afluente de militantes provenientes de Francia y España, recién en 1884 tenemos noticias de la existencia de un grupo llamado "Círculo Comunista Anárquico, sección de la AIT" del cual participaron Ettore Mattei, Marino Gabaccio, Miguel Fazzi y Washington Marzorati, entre otros.

Al año siguiente, en 1885, no sólo se inicia el inaugural periódico de Malatesta La Questione Sociale (1885-1886) y su "Círculo de Estudios Sociales", sino que también se instala en Buenos Aires la "Librería Internacional" de Emile Piette, que sería el primer punto de distribución de folletería ácrata francesa, italiana y española ${ }^{4}$. Recién en 1886, probablemente, la publicación periódica La lucha obrera editó los dos primeros folletos libertarios aquí impresos 5 .

\footnotetext{
${ }^{2}$ Las cartas de Juanes y Galcerán, fechadas respectivamente el 7/4/1872 y el 25/5/2872, fueron recuperadas por José Valadés en "Documentos para la historia del anarquismo en América", Certamen Internacional de La Protesta, Buenos Aires, La Protesta, 1927. Hay que tener en cuenta que, mientras tanto, ese mismo año se fundaba en Montevideo una seccional de fuerte impronta libertaria que en 1875 había editado un primer folleto y dos años después contaba con 2000 miembros. Ver: Zaragoza Ruvira, Gonzalo, Anarquismo argentino (1876-1902), Madrid, Ediciones de la Torre, 1996. Sobre la correspondencia de Wilmart y los primeros episodios de la Internacional en Argentina sigo: Tarcus, Horacio, Marx en la Argentina, Buenos Aires, sigloXXI, 2007.

3 Ingenieros, José, "La Internacional en Sud América: datos que servirán para la historia del socialismo", Almanaque socialista de La Vanguardia, Buenos Aires, 1899.

${ }^{4}$ Al menos en Buenos Aires, es a partir de 1897 cuando comienza a producirse un aumento escalonado en la aparición de librerías. Hasta 1890 no existía un circuito específico de circulación del libro y tan solo podían encontrarse algunas pocas librerías en Buenos Aires. Es por eso que, en un momento donde los libros se vendían en casas de distintos rubros, se trata de un muy temprano establecimiento de este tipo. Cuatro años después esta librería sería allanada y Piette encarcelado por ocho meses. En 1895, tras su fallecimiento, su librería pasará a ser atendida por el militante anarquista Alex Sadier.

${ }^{5}$ Existen diferentes versiones sobre el lugar y año de edición de este periódico. Para algunos fue publicado en 1882, para otros en 1885 o 1886. Tampoco queda claro si apareció en Buenos Aires o Montevideo. Según varias fuentes, los dos folletos hoy perdidos que editaron desde este medio fueron: Esteve, Pedro, La mujer,
} 
Una vez cerrada La Questione Sociale, durante 1887 Ettore Mattei buscó continuar la labor propagandística de este periódico con los ocho números gestados desde la Sociedad de Obreros Panaderos que aparecieron de Il socialista. A partir de los anuncios de sus páginas se pueden rastrear los títulos de los primeros folletos que circularon por Argentina desde la redacción de este periódico, y que consistieron principalmente en prensa y folletería editada en Italia. Por esta vía llegaron autores como Malatesta, Cafiero, Kropotkin, Merlino, Pistolessi y Castellazo; y los periódicos italianos Humanitas, La lotta, La Montagna y Paupertas, La Révolte de Paris, El productor de Barcelona y la Revista social de Madrid. Cuando, entre 1888 y 1889, comenzó el primer ciclo de grandes huelgas en la Argentina, esta misma sociedad de panaderos participó de una edición conjunta de una página publicada en ambas costas del Río de la Plata con formato periódico y titulado Undice Novembre en conmemoración por los anarquistas ahorcados en Chicago tras la revuelta de Haymarket; y es gracias a este impreso que es posible conocer la existencia de al menos cinco grupos libertarios en Buenos Aires hacia $1889^{6}$.

\section{Auge de las prácticas editoriales del anarquismo argentino (1890-1901)}

Sin duda el gran crecimiento de publicaciones libertarias se daría en la década siguiente que se abre con la aparición de los periódicos La miseria (1890-1891) y El perseguido (18901897) en momentos de una fuerte crisis económica y política poco después de que mermaran los créditos ingleses y el Estado argentino se declarara en bancarrota. Suele afirmarse que a partir de La questione sociale de 1885 hubo un primer ciclo de publicaciones anarquistas conformadas según comunidades idiomáticas, con sus correspondientes periódicos en italiano — como Lavoriamo (1893), Demoliamo (1893) y su continuación La Riscossa (1893 y 1894), Il paria (Rosario, 1899) y, más tarde, Nuova Civilitá (1901-1902) y L'Agitatore (Buenos Aires, 1904) - y aquellos del efímero grupo francoparlante, La liberté (1893-1894) y su continuador Le Cyclone (1895-1896) ${ }^{7}$.

[Buenos Aires], La Lucha Obrera, [1886]; y ¿Qué es la solidaridad?, [Buenos Aires], La Lucha Obrera, [1886]. Después, ese mismo año, siguiendo la bibliografía de Santillán, en 1886 también se publicó en forma de folleto un manifiesto comunista-anárquico en Buenos Aires. Luego otro en 1888 que hacía referencia a los llamados Mártires de Chicago. Y otro en 1889 en el contexto de las huelgas de carpinteros y albañiles. Ninguno de estos se ha conservado.

${ }^{6}$ Por este periódico sabemos que, además de la Sociedad de Obreros Panaderos, al finalizar la década había una serie de grupos libertarios ya conformados: 11 de Noviembre, Círculo Socialista Internacional, Los Miserables, Luisa Michel, y Juventud Revolucionaria de Montevideo. Por otra parte, desde 1882 ya se había desarrollado una serie de periódicos socialistas más estables: Vörwarts (1886-1901), El Obrero (1890-1892; 1893) — órgano de la Federación Obrera de orientación socialista que dura hasta 1897_, El Socialista (1893), La Vanguardia (1894-publicación abierta), y La Rivendicazione (1896).

${ }^{7}$ A diferencia de lo que sucedió en otras corrientes políticas, es notorio que siguieron editándose periódicos libertarios e incluso algunos folletos desde una determinada comunidad lingüística en italiano, ruso o idish hasta bien entrado el siglo XX. Entre ellos: dos periódicos más titulados La Rivolta, uno de 1917 y otro publicado entre 1924 y 1925; el grupo de publicaciones de las que participan y/o impulsan Aldo Aguzzi y Severino di Giovanni: L'avvemire (1923-1925), Culmine (1925-1928), Anarchia (1930); y el ciclo de publicaciones rusas alrededor de Anatol Gorelik, Golos Truda (1917-1930), Anarchija (1930), голос из подполья [Una voz desde el subsuelo] (1930) у Vol 'Naia Mysl' [Pensamientos libres] (1932); a lo que hay 
Estas publicaciones libertarias resultaron en definitiva más breves y discontinuas que aquellas que, también con columnas en varios idiomas, fueron impulsadas por grupos e individuos según su afinidad ideológica y lograron durabilidad marcando los lineamientos iniciales dentro del campo anarquista: nos referimos a El Perseguido, en primer lugar, y en un segundo momento a El Oprimido (1893-1897), L'Avvenire (1895-1904), La Protesta Humana (1897-1904) y El Rebelde (1898-1903). En este sentido, con sus 102 números, El perseguido, primero editado por el Grupo "Los Desheredados" y después por "La Expropiación", tuvo un papel preponderante en la escena ácrata. Este periódico generó el campo de vínculos y discusiones iniciáticas, e incluyó notas firmadas por los principales militantes libertarios que estuvieron por Buenos Aires durante aquellos años, como Rafael Roca, Baldomero Salbans, Manuel y José Reguera, Pierre Quiroule, Jean Raoux, Francisco Denambride, Santiago Locascio y Orsini Menoti Bertani ${ }^{8}$.

Según narra Gilimón en 1911, la edición de folletos constituyó desde el comienzo un objetivo primordial que se enfrentaba a dos límites: el dinero y la falta de traducciones. Pero, sostiene, que al igual que las conferencias, picnics y periódicos, los folletos que se editaban tenían muy buen recibimiento y repercusión, según este autor, debido a la falta de ofertas culturales populares que había en ese entonces ${ }^{9}$. Mientras los periódicos cumplían principalmente una función propagandística y constituían un instrumento de organización y comunicación, los folletos contenían una preocupación teórica centrada en ampliar ciertos temas que se difundían fragmentariamente en los periódicos y constituían el objeto específico de algunas revistas libertarias. Por lo que este dispositivo constituido por el periódico y su serie monográfica de folletos conformaron sin duda un medio de difusión con ciertas características propias — en cuanto a su proyecto, financiación y distribuciónque hacen sobresalir a la actividad editorial libertaria dentro de la historia del libro local.

Si bien surgieron algunos grupos que principalmente buscaron dedicarse a la edición de folletos, como el Grupo Juventud Comunista-Anárquica (1892-1895) y Los Ácratas (1897-1899), por lo general los primeros proyectos editoriales fueron parte de algún grupo que a la par editaba también una publicación periódica - lo que era habitual también en la prensa comercial de la época— ${ }^{10}$. Por ejemplo, los ya nombrados periódicos La questione

que sumarle la publicación íntegramente en idish Dos Fraie Vort [La libre palabra] [1920-1967]. Tanto Golos Truda como Culmine y Anarchia editaron folletos y libros en ruso e italiano entre 1923 y 1927.

${ }^{8}$ También a partir de este periódico se delinean las distintas trayectorias posteriores. Hacia 1894 Fortunato Serantoni rompe por diferencias ideológicas con El perseguido y comienza su propio periódico y editorial $L a$ questione sociale (1894-1896), que junto a El oprimido y al año siguiente $L$ ' Avvenire buscarán continuar la línea organizadora iniciada por Malatesta en la década anterior. Por otra parte, Bertani junto a otros militantes en 1896 pasó a constituir el grupo de redactores de la publicación La revolución social (1896-1897) que poco antes había sido fundada por Manuel Reguera y Gregorio Inglán Lafarga. Una vez cerrado El perseguido en 1897, es Baldomero Salbans, director de sus suplementos, quien busca proseguirlo en su emprendimiento $L a$ Autonomía (1897). Aunque, acorde a los objetivos declarados en su primer número, será El rebelde su más perdurable continuador.

9 Gilimón, Eduardo, Hechos y comentarios, Montevideo, [s.n.], 1911.

${ }^{10}$ Específicamente sobre la prensa anarquista y un análisis de distintos aspectos alrededor de ella: Anapios, Luciana, "Una promesa de folletos. El rol de la prensa en el movimiento anarquista en la Argentina (18901930)", A Contracorriente $\mathrm{n}^{\circ}$ 2, vol. 8, 2011; Fernández Cordero, Laura, "Un ejercicio de lectura sobre el concierto de la prensa anarquista a partir de Mijail Bajtin (Argentina, 1895-1925)", Adversus X, 2013. 
sociale, El Rebelde, L'Avvenire y La Protesta buscaron publicar sus propios folletos. Debido a ello, inicialmente también una de las primeras maneras de editar textos largos por parte de los grupos de propaganda fue su publicación por entregas en el correr de los números de un periódico ${ }^{11}$.

En 1895 se publican los que Max Nettlau considera los dos primeros libros anarquistas en toda América. Uno de ellos es La conquista del pan de Kropotkin traducido del francés con un prefacio de Eliseo Reclús y editado por La Expropiación y $E l$ Perseguido; y el segundo — editado por la Imprenta Elzeviriana de Pedro Tonini- es el de Jean Grave La sociedad moribunda y la anarquía; en ambos casos se destacan del resto de los folletos únicamente por tratarse de unas casi 200 páginas abrochadas por un gancho de metal al medio. A partir de este último título, el tipógrafo italiano Pedro Tonini comenzó su propia "Biblioteca de Estudios Sociales" e imprimió otros títulos a pedido de distintos emprendimientos, como la Biblioteca de La Questione Sociale y la del grupo Los Ácratas, y periódicos como L'Avvenire y Ciencia Social, además de algunos materiales socialistas. El mismo grupo que se encargó de la edición de El perseguido durante algunos años — "La Expropiación Grupo de propaganda anárquica"-emprendió durante 1895 la edición de una serie de folletos llegando a editar seis títulos; todos folletos entre 16 y 32 páginas que salieron con una tirada de 3000 ejemplares. Por su parte, durante 1895 y 1896 el militante italiano Fortunato Serantoni impulsó a la par de su periódico La questione sociale una "biblioteca" del periódico, que constó de ocho títulos, entre los cuales cinco son parte de la colección "Propaganda anárquica entre las mujeres"12. Este emprendimiento será continuado por Serantoni en la colección de la Librería Sociológica que editó dieciséis folletos antes de que su mentor sea expulsado del país en 1902. También cabe destacar la labor editorial del grupo Los Ácratas que participó de la edición de El Rebelde y editó doce folletos en cuatro años en tiradas que alcanzaban los 10000 ejemplares. Y también, para nombrar las iniciativas de mayor duración de estos años, la biblioteca rosarina Ciencia y Progreso, al mismo tiempo que sacaba el periódico La nueva humanidad, llevó a cabo la edición de las conferencias de Emilio Arana, quien de esta manera se convirtió en el primer autor argentino en ser publicado en ediciones monográficas anarquistas.

En todos estos casos se trató siempre de títulos editados por primera vez en Argentina en formatos pequeños con el objetivo de lograr un bajo costo unitario. Tanto los folletos como los periódicos generalmente se financiaban por suscripción previa, y en

\footnotetext{
${ }^{11}$ De esta manera se editaron principalmente textos de Eliseo Reclús y Kropotkine, quienes durante las siguientes décadas se mostrarían como los autores canónicos en el anarquismo argentino. Respecto a Reclús, el periódico El Perseguido editó “¿Por qué somos anarquistas?”; La Liberté en 1894 publicó en sus páginas dos textos de Reclús en francés, "A mon frére le paysan" y "La riqueza y la miseria", que quedó inacabado; en 1895, La Questione Sociale publicó por capítulos L’ Anarchie en italiano. Y algo similar ocurrió con los textos de Kropotkine que aparecieron por partes tanto en italiano, francés y español, nuevamente en El Perseguido, La Liberté y La Verdad de Rosario. Además, otro género que fue editado de esta manera fueron las declaraciones de militantes anarquistas frente a los tribunales: la de Clement Duval en La Libre Iniciativa, Rosario, 1896; Ravachol en El Perseguido, 1892; Salvador Franch en El Perseguido, 1995; Emile Henry (1894) en El Oprimido.

${ }^{12}$ Existe una edición facsimilar reciente de algunos de los folletos de La Expropiación y La Questione Sociale: Ferrer, Christian - Albornoz, Martín (Selección y Prólogo), Folletos anarquistas en Buenos Aires, Buenos Aires: Biblioteca Nacional, 2015.
} 
menor medida mediante conferencias y veladas culturales. Pero en ningún caso se publicaba para financiar otros proyectos. Por el contrario, se realizaban distintas actividades con el fin de recaudar fondos para proseguir la tarea editorial, de manera que la divulgación de ideas funcionaba como un fin último. Cuando aparecía el folleto publicado, en su última página constaba la lista de suscriptores que lo habían hecho posible y a su vez anticipaba el próximo título a editar para promover un nuevo proceso de recaudación. Incluso algunos traían una última hoja para arrancar, la cual constaba de una grilla para apuntar nombre y monto de cada suscriptor y que luego podía ser remitida a la redacción de algunos de los periódicos anarquistas en curso; con lo cual se convocaba a que cualquier lector fuera también un potencial recaudador de dinero para el proyecto editorial.

De esta manera no sólo se buscaba descentralizar la financiación sino también la circulación, ya que al mismo tiempo se invitaba a pedir una determinada cantidad de folletos a quienes quisieran repartirlos. Como resume desde su tapa uno de ellos, por lo general el objetivo fue que cada folleto fuera "editado por contribución voluntaria y repartido gratuitamente". Lejos de ser pensados con la expectativa de una inversión en las coordenadas de la oferta y la demanda, estos emprendimientos contienen su propio límite en su público que está llamado entonces a sostenerlos materialmente, e incluso en algunos casos a participar del propio proceso de circulación ${ }^{13}$.

A partir de 1901, con la estabilización de los proyectos propagandísticos de Ciencia social, L' Avvenire y La Protesta y el asentamiento de La Protesta y Fueyo como las editoriales libertarias de mayor envergadura y duración, la prensa organizadora logró promover una serie de iniciativas que terminarían en la conformación de la FORA. Mediante esta federación gremial los militantes libertarios se mostrarán protagonistas del ciclo huelguístico que comenzará con el siglo y no concluirá hasta el estado de sitio de fines de 1902 y la correspondiente promulgación de la Ley de Residencia ${ }^{14}$.

\section{Bibliotecas, librerías, revistas y folletos anarquistas: politizar la opresión cotidiana}

Desde el ya mencionado caso inicial de Il socialista, las redacciones de los periódicos libertarios constituyeron un punto de distribución habitual de la folletería

\footnotetext{
${ }^{13}$ Por ejemplo esto queda claro al leer la advertencia del primer número de la revista santafesina La campana (1919): "Lector amigo: Los primeros números de esta revista son de prueba, para pulsar la opinión y hacer el intento de reanudar nuevas amistades y relaciones. En primer término, se quiere comprobar si hay suficiente número de interesados para una revista de esta especie, cuyo formato, precio y características definitivas se fijarán una vez hecha la consulta debida a los lectores".

${ }^{14}$ Para un análisis exhaustivo de este proceso, las referencias oblligadas a los trabajos de Oved, Zaragoza y Suriano permiten adentrarse en las discusiones culturales y gremiales del anarquismo de este período. Ver: Oved, Iacov, El anarquismo y el movimiento obrero en Argentina, México, Siglo XXI, 1978; Zaragoza Ruvira, Gonzalo, Anarquismo argentino (1876-1902), Madrid, Ediciones de la Torre, 1996. Suriano, Juan, Anarquistas, Buenos Aires, Manantial, 2001.
} 
anarquista local e internacional ${ }^{15}$. Muchas veces estas redacciones funcionaban a la vez como librería y espacio de discusión y lectura, en paralelo a las pocas librerías libertarias propiamente dichas, que no resultaban menos polifuncionales. Al menos entre 1896 y 1901 la nombrada Librería Sociológica de Fortunato Serantoni, además de vender libros y folletería internacional, en la práctica funcionó como biblioteca, redacción de las revistas La questione sociale y Ciencia Social y como centro medular del anarquismo porteño, donde se recibían los dineros de las suscripciones para distintos periódicos y folletos. Cuando ésta fue destruida por la represión policial en 1901, Serantoni tuvo que escapar a Montevideo y la Librería Sociológica de Bautista Fueyo se mostró como su mejor reemplazo por varios años ${ }^{16}$.

Otro punto de difusión importante fueron las nacientes bibliotecas obreras. Desaparecida en 1876 la Comisión Protectora de Bibliotecas Populares creada por Domingo Faustino Sarmiento, se dio una vacancia estatal sobre políticas bibliotecarias que en buena medida fue aprovechada y suplida por asociaciones gremiales socialistas y anarquistas $^{17}$. La creación de bibliotecas se presentó como un objetivo prioritario para distintos grupos anarquistas que iba de la mano con las políticas editoriales y la promoción de escuelas libertarias. Explícitamente fue a partir de 1903, cuando en el congreso de la FORA de ese año se comenzó a recomendar a las distintas sociedades de resistencia la creación de bibliotecas aledañas ${ }^{18}$. En una costumbre que se sostendrá por décadas, para

${ }^{15}$ Casi todas las redacciones poseían un servicio de librería que ofrecía ediciones locales y extranjeras publicitadas en cada periódico. A través sus distintos catálogos es posible rastrear la disponibilidad de los productos editoriales de proyectos libertarios de Uruguay, España e Italia, y en menor medida de Francia, Estados Unidos y Chile. Entre aquellos proyectos de ediciones monográficas extranjeras que circulaban por Buenos Aires hasta 1905, podemos nombrar al menos los siguientes. Proyectos editoriales españoles: El Productor (Barcelona), Biblioteca Juventud Libertaria (Barcelona), El Progreso (La Coruña), Biblioteca Ácrata (Barcelona), Biblioteca de La Huelga General (Madrid), Biblioteca Anarquista (Madrid). De Italia: Biblioteca Popolare Educativa (Torino), Il Pensiero (Roma), Biblioteca Di Propaganda del Circolo Di Studi Sociali Corticellese (Bologna), Tipografìa Cooperativa Sociale (Roma), Biblioteca Di Propaganda el Circulo Studi Sociali (Padova). Franceses: las ediciones del Groupe des Étudiants Socialistes Revolutionnaires Internationalistes (Paris); La Révolte (Paris), Temps Nouveaux (Paris), Bibliothèque Anarchiste (Paris). De Estados Unidos: Biblioteca de El Despertar (Brooklyn), Librería Sociológica (Paterson, NJ), Biblioteca Anarchica Dell' Aurora (West Hoboken, NJ). De Uruguay: Grupo Anárquico de Montevideo; Grupo Aurora (Montevideo); Centro Internacional de Estudios Sociales (Montevideo); Biblioteca de La Rebelión (Montevideo). Y de Chile, la Biblioteca de El Martillo (Santiago).

${ }^{16}$ La conocida Librería Sociológica de Fortunato Serantoni estuvo ubicada en Corrientes 2039/41. Por su parte, en un principio la librería-imprenta de Fueyo funcionó sobre lo que es hoy Avenida 9 de Julio cerca de Retiro. Luego, durante la década del diez, en Azcuénaga 16 y luego en Pavón 2860.

${ }^{17}$ Sobre la historia de las bibliotecas argentinas: Sarmiento, Nicanor, Historia del Libro y de las Bibliotecas Argentinas, Buenos Aires, Imp. Luis Veggia, 1930; Tripaldi, Nicolás, "Origen e inserción de las bibliotecas obreras en el entorno bibliotecario argentino", Libraria 1, Buenos Aires, 1997; Sik, Eugenia, "La creación de bibliotecas durante el apogeo del anarquismo argentino (1898-1905)" [mimeo] [en prensa].

${ }^{18}$ Siguiendo el recién citado texto de Eugenia Sik, es a partir de la nombrada disposición realizada por el congreso de la FORA de 1903 cuando se registra un aumento aún mayor de bibliotecas vinculadas a las distintas sociedades de resistencia. Este texto además rastrea en periódicos anarquistas y socialistas las críticas a las restrictivas condiciones de consulta que poseía la Biblioteca Nacional y a la discrecionalidad de sus adquisiciones bibliográficas, por lo que — además de constituirse como un espacio de sociabilidad política- 
ampliar el alcance de su difusión, los folletos mismos llamaban a no ser conservados en bibliotecas personales. Y tempranamente encontramos inscriptas en la tapa de distintas publicaciones frases como "Prestad un servicio a la humanidad, haciendo circular este folleto entre vuestras amistades"; o directamente: "Dijimos y volvemos a repetir: la propiedad es un robo; quien retiene para sí esta hoja, es un ladrón... iqué circule!"19.

Para dimensionar esta última frase posiblemente ahora nos resulte difícil comprender el aura involucrada en estos libros que eran considerados auténticas herramientas de transformación por parte de sus editores. Una de las formas de intentar vislumbrarlo podría ser transcribir aquellos parágrafos de los memoriales militantes tanto socialistas como anarquistas donde sus autores dan cuenta de la transformación súbita que les provocó la lectura de un determinado texto mediante el cual "la Idea" estableció un antes y un después en sus vidas. Otra de las maneras de comprenderlo consiste en mirar los títulos editados y observar que el conjunto de intereses y temas — que en gran medida se mantienen hasta hoy - constituyen luchas prácticas de comportamiento y superación individual que desde el anarquismo se entendieron de una preponderancia política fundamental frente a distintas formas de opresión. La mayoría de ellos, por su temática y contenido -emancipación sexual, anticlericalismo, antimilitarismo, antipatriotismo, naturismo, educación y lucha obrera-, promovían una politización inmediata de las prácticas cotidianas. Precisamente, en la declaración de sus proyectos, todas estas iniciativas editoriales sostenían que buscaban "causar una revolución moral en los lectores" desde una concepción fuertemente iluminista del libro como instrumento de educación y emancipación, que pretendió generar lectores "librepensadores" con los ánimos de combatir la mecánica educativa aleccionadora de la escuela pública y el servicio militar como modelo de asimilación de extranjeros ${ }^{20}$.

Al respecto, hay que remarcar que estos folletos fueron parte de un conjunto de actividades como la lectura pública comentada y las conferencias, y constituyeron un círculo más amplio de prácticas entre el periódico, la biblioteca y el gremio ${ }^{21}$. Y es en el conjunto de esta sociabilidad política en la cual hay que comprender el surgimiento de esta literatura libertaria dentro del proceso más amplio de constitución de un campo cultural

las bibliotecas obreras buscarían diferenciarse sustancialmente en sus horarios de apertura y el material de consulta disponible.

${ }^{19}$ En Prometeo $\mathrm{n}^{\mathbf{0}}$ 1, agosto 1919; en 1925, el grupo editor Regeneración encabezaba la tapa de su folleto de la misma manera: "La propiedad privada es un robo. Quien después de leer este folleto se lo guarde es un ladrón".

${ }^{20}$ Contra la posibilidad de que la lectura militante sea una lectura ilustrada, el análisis criminológico que hace Francisco de Veyga del militante Salvador Planas critica el conocimiento de textos que posee el condenado, porque éste se muestra mecánico y repetitivo, producto de su lectura dogmática que "no alcanza a saborear la lectura ni menos a comprender la especiosa argumentación de Eliseo Reclus, las brillantes utopías de Kropotkine...”. En este texto Veyga sugiere la siguiente distinción: por un lado el anarquismo editó obras sencillas de escaso valor (como las de Grave, Malatesta y Malato), mientras, por otro, textos de mucha más difícil e inaccesible lectura. Ver: de Veyga, F., "Delito Político: El anarquista Planas Virella", Archivos de psiquiatría y criminología V, Buenos Aires, 1906. Para un análisis amplio de las prácticas de lectura anarquista puede verse: Di Stefano, M., El lector libertario, Buenos Aires, Eudeba, 2013.

21 Ver: Barrancos, D., "Las 'lecturas comentadas': un dispositivo para la formación de la conciencia contestataria entre 1914-1930", Boletín CEIL XVI, 1987. 
moderno y la ampliación del público lector. En este marco, los objetivos emancipatorios de estas lecturas resultaron pioneros en intentar difuminar las diferencias entre un circuito culto y otro popular antes del $900^{22}$.

Efectivamente el bajo precio y la interpelación política amplía - tanto en español como en italiano - que promovían estos textos hayan dado una difusión destacable al impreso anarquista ${ }^{23}$. Como dijimos, hasta ese momento las "ediciones populares" existentes publicaban únicamente obras literarias. En 1901 comenzó "La biblioteca del diario La Nación” que se extendió hasta 1920. Recién en 1915 José Ingenieros y Ricardo Rojas lanzan sus respectivas colecciones editoriales dedicadas a autores nacionales, respectivamente, La Cultura Argentina y La Biblioteca Argentina; entre ellas únicamente la primera buscaría resultar económica para lograr un alcance masivo. De todas maneras, en ningún caso estas colecciones tomaron específicamente como interlocutores a trabajadores, “oprimidos y proletarios". En contraposición, las iniciativas editoriales socialistas funcionaron de manera mucho más centralizada alrededor de La vanguardia especialmente desde 1905- y únicamente publicaron textos programáticos y en castellano, buscando una identidad de la clase obrera y la institucionalización del partido ${ }^{24}$. De manera que, en este marco, las ediciones libertarias fueron las primeras que buscaron un alcance económico y a la vez una interpelación política amplia no programática ${ }^{25}$.

Después de 1901 las colecciones de folletos anarquistas se volvieron discontinuas ${ }^{26}$. Sus luchas y temáticas fueron desarrolladas por un nuevo formato de revistas "culturales"

\footnotetext{
${ }^{22}$ Para pensar la ampliación del público lector en relación al nuevo marco editorial de estos años resulta imprescindible Prieto, Adolfo, El discurso criollista en la formación de la Argentina moderna, Buenos Aires, Sudamericana, 1988

${ }^{23}$ En 1909, desde el Ministerio de Instrucción Pública, Ricardo Rojas trazaba una alarmada retrospectiva sobre el peligro que generaron estos "ávidos editores", ya que habían logrado que "peligrosa filosofía circulase en volúmenes económicos, más asequibles que el libro nacional y los manuales de escuela" ( $L a$ restauración nacionalista, Buenos Aires, Ministerio de Instrucción Pública, 1909, p. 38). Otra forma de dimensionar el alcance de esta producción nos las da Diego Abad de Santillán. Según este autor, hacia fines del siglo XIX, el mercado de folletería anarquista local permitía sostener iniciativas editoriales libertarias provenientes de Italia, España y Uruguay, mientras que a los proyectos editoriales locales les alcanzaba con la demanda inmediata (Ver: El anarquismo en el movimiento obrero, Barcelona, Cosmos, 1925).

${ }^{24}$ Dentro de la oferta que hacía la izquierda en lo que respecta a la edición de folletos, por su parte, el movimiento sindicalista revolucionario solamente desarrolló cinco o seis series monográficas que repitieron entre sí muchos de sus títulos: El Obrero (1906), La Acción Obrera (1913-1918), Agrupación sindicalista [c. 1920] y el Sindicato de Obreros Ebanistas (1920-1921). A éstas hay que sumarle los folletos editados por el Consejo Federal de la llamada FORA-IX, la Unión Sindical Argentina y Bandera Proletaria. Sobre los emprendimientos editoriales socialistas, ver: Tarcus, H., Marx en la Argentina, Ibid., y Buonnome, J., "Cultura impresa y socialismo. Lecturas sobre la historia de la prensa socialista en tiempos de la Segunda Internacional", Políticas de la memoria 14, 2012-2013. Para un análisis de las discusiones entre socialistas y anarquistas a través de la prensa: Albornoz, M., "Caleidoscopio de palabras. Las reuniones de controversia entre anarquistas y socialistas a finales del siglo XIX y principios del siglo XX”, Sociedad 28, 2009.

${ }^{25}$ Sobre las colecciones editoriales del entresiglo argentino sigo: Degiovanni, F., Los textos de la patria. Buenos Aires: Beatriz Viterbo, 2007; Pastormerlo, S., "El surgimiento de un mercado editorial”, en De Diego, J. L. (dir.), Editores y políticas editoriales en Argentina, Ibid.

${ }^{26}$ Efectivamente los distintos grupos dejan de editar folletos seriados y aquellos que lo intentan sólo logran publicar uno o dos títulos corridos. Esto es visible en la cantidad de folletos anarquistas publicados. Aquí los datos según año y cantidad de folletos impresos. 1895: 10 títulos; 1896: 8; 1897: 15; 1898: 12; 1899: 12;
} 
que, en conjunción con la serie de revistas anarquistas literarias, se extiende al menos hasta fines de la década del veinte ${ }^{27}$. De hecho, con el fin de otorgarles a estos temas mayor especificidad y espesor, desde temprano aparecieron una gran cantidad de revistas y periódicos que se encargaron de ampliar y desarrollar ciertas luchas específicas presentes en todos los periódicos libertarios. Corriéndose de la intervención directamente coyuntural, los intereses temáticos que solían ocupar la edición de folletos tuvieron entonces también su manifestación en revistas particulares que tomaron de manera central la lucha por la emancipación sexual, el librepensamiento, el antimilitarismo o el naturismo, y desde el desarrollo de uno de estos temas desplegaban las demás batallas que promovía el anarquismo $^{28}$. En contrapartida, entre 1902 y 1909, se multiplicaron las publicaciones periódicas gremiales. Como señaló Bilsky, entre 1901 y 1906 se registró la mayor cantidad de creación de entidades gremiales y al mismo tiempo la mayor cantidad de afiliaciones a la FORA y a la UGT (Unión General de Trabajadores, impulsada por los socialistas y, a partir de 1906 , por los sindicalistas $)^{29}$.

A la par de las revistas literarias del anarquismo afloraron pequeñas colecciones de uno o dos folletos sobre teatro y poesía, muchas veces también asociadas a periódicos obreros. Pero la actividad editora libertaria menguará considerablemente a partir de 1910, cuando, en vísperas de la implementación de la Ley de Defensa Social, se incendiaron tanto las imprentas de La Protesta como la de Fueyo. La producción de impresos libertarios recién volvería a dispararse a fines de la década con la llegada de noticias sobre la Revolución Rusa.

1900: 12; 1901: 10; 1902: 6; 1903: 2; 1904: 4; 1905: 7; 1896: 5; 1907: 11; 1908: 3; 1909: 10; 1910: 5; 1911: 3; 1912: 3: ; 1913: 1; 1914: 7; 1915: 3; 1916: 3; 1917: 1 .

${ }^{27}$ Hacia 1897 nace un género de revistas libertarias anarquistas que constituirá un medio de prensa fundamental en varios aspectos. Ciencia social (1897-1898) de Fortunato Serantoni abre todo un ciclo de revistas culturales que llega hasta el Suplemento de La Protesta editado entre 1922 y 1930. Una vez cerrada Ciencia Social, revistas como El Sol (1898-1903) de Alberto Ghiraldo, El Escalpelo (1899-1900), Tiempos nuevos (1900) de Félix Basterra, Martín Fierro (1904-1905) también de Ghiraldo, Los nuevos caminos (19061907) y Letras (1907) —las dos de José de Maturana-, las revistas de Alejandro Sux Germen (1906-1911) y Ariel (Paris, 1912-1913), Ideas y figuras (1909-1916,1918-1919), Estudios (Rosario, 1915-1916) у La campana (Santa Fe, 1919), entre otras, crearon un periodismo militante previo a la profesionalización cultural de Buenos Aires. De manera tal que la colaboración en estas revistas libertarias resultó ser una de las maneras de ingresar al circuito periodístico y cultural del momento.

${ }^{28}$ Como dijimos al comienzo, la propia historiografía de la izquierda se enfocó en la conformación del movimiento obrero mientras brindaba una importancia secundaria a la presencia de "otras" temáticas consideradas meramente culturales, sin poder valorar entonces gran parte de la apuesta política del anarquismo como movimiento. Al igual que los folletos, con el fin de otorgarles a estos temas mayor especificidad y espesor, desde temprano aparecieron una gran cantidad de revistas que se encargaron de desarrollar estas luchas específicas y tomaron de manera central la lucha por la emancipación sexual, — La voz de la mujer (Buenos Aires, 1896-1897) y La voz de la mujer (Rosario, 1899-1900) —, el librepensamiento La fuerza de la razón (1896) - el antimilitarismo — Luz al soldado (1907-1914), El Cuartel (1909)—, el naturismo - La renovación (1902) - y la educación libertaria - La escuela moderna (1908-1909), Francisco Ferrer (1911-1912); por nombrar sólo algunas.

${ }^{29}$ Bilsky, E., La FORA y el movimiento obrero, Buenos Aires, CEAL, 1985. Durante los mismos años, los fuertes ciclos huelguísticos de 1904-1905, 1906-1907, 1908-1909 se correspondieron con un aumento en la cantidad de deportados por aplicación de la Ley de Residencia. 


\section{Folletos y libros anarquistas de la década del 20: una cultura libertaria}

De acuerdo con el trabajo de Doeswijk, entre 1918 y mediados de 1921 casi todo el arco del anarquismo local apoyó la Revolución Rusa ${ }^{30}$. Al calor de las distintas discusiones internas, en estos años surgirá una nueva cantidad de efímeros grupos editores de periódicos y folletos que apuntaron a intervenir de distinta manera dentro del Partido Socialista, el Partido Socialista Internacional, la FORAV y la FORAIX. Efectivamente, la lista de emprendimientos editoriales se multiplica durante estos años: Adelante, La Internacional, Editorial Marxista, Pax, Spartacus, Regeneración, Agrupación sindicalista, Liga de educación racionalista, Los comunistas, Ángel Zuccarelli y Argonauta, entre otras.

Dentro del campo específicamente anarquista, la mayoría de los folletos publicados emprenden una discusión sobre la necesidad de renovación del programa libertario. Mientras, por un lado, algunos sectores promovieron un nuevo grupo de influencias y referencias culturales ligadas al bolchevismo, por otra parte, especialmente a partir de 1921, otros insistirán en la necesidad de plegarse más firmemente sobre sus bases y trazar nuevas diferenciaciones con el sindicalismo revolucionario y la línea revolucionaria bolchevique dentro del socialismo. De manera que, entre 1919 y 1923, apareció todo un nuevo conglomerado de textos que pujaron por lograr distintas definiciones identitarias dentro del movimiento anarquista según programas de militancia en buena medida rivales ${ }^{31}$.

Pasado el denominado "trienio rojo argentino", durante la década del veinte aparecen dos importantes novedades en la serie de las ediciones monográficas anarquistas. Por un lado surge un nuevo conjunto de folletines de literatura, pensamiento, poesía y teatro que conforman algo así como una cultura libertaria que apunta a un amplio humanismo universal y en buena medida se vuelve común al arco de las izquierdas. Para ampliar su llegada hacia más lectores, sus publicistas - Julio Barcos, Bautista Fueyo y Fernando Gualtieri- dirigen su actividad editora a un nuevo género intermedio entre el folleto y la publicación periódica: los folletos seriados, que circulaban en Buenos Aires desde 1917 y, dentro de la izquierda, habían sido inaugurados en 1921 por Antonio Zamora y su colección Los Pensadores. De aquí en más, estos emprendimientos de tirada amplia y barata sí estarán dirigidos específicamente al "pueblo trabajador"32. En primer lugar se destacan las

\footnotetext{
30 Sobre este período resultan fundamentales dos trabajos: Doeswijk, Andreas, Los anarcobolcheviques rioplantenses, Buenos Aires, CeDInCI, 2013; Pittaluga, Roberto, Soviets en Buenos Aires, Buenos Aires, Prometeo, 2015.

${ }^{31} \mathrm{Al}$ continuar el relevamiento iniciado en la nota 25 , vemos que sólo dentro del anarquismo entre 1919 y 1924 se publicaron más de 250 títulos en formato de pequeños folletos, según año y cantidad de títulos: 1918: 10 títulos; 1919: 13; 1920: 24; 1921: 27; 1922: 55; 1923: 81; 1924: 43; 1925: 17; 1926: 18; 1827: 26; 1928: 23; 1929: 18; 1930: 16.

${ }^{32}$ Como señalan Gutierrez y Romero en su inaugural texto sobre el tema, probablemente puede hablarse de una segunda expansión del publico lector durante estos años. Estos autores proponen también pensar estos emprendimientos como empresas culturales - y no como específicamente comerciales - teniendo en cuenta el trabajo social que se proponían. Ver: Gutierrez, Leando y Romero, José Luís, "Buenos Aires en la entreguerra: libros baratos y cultura de los sectores populares", en Diego Armus (comp.), Mundo urbano y cultura popular: Estuidios de historia social, Buenos Aires, Sudamericana, 1990; Romero, José Luís, "Sociedades barriales, bibliotecas populares y cultura de los sectores populares: Buenos Aires, 1920-1945", Desarrollo económico 113, 1989.
} 
colecciones La Palestra (1922-1929) de Gualtieri y Sembrando Ideas (1923-1925) de Fueyo, que fueron de una indudable inscripción anarquista, y que se suman a la importante difusión teatral que Fueyo venía realizando desde su sello. Pero también al mismo tiempo aparece Las grandes obras (1922-1924) encabezada por Barcos, que combina autores libertarios, escritores rusos hasta ese momento desconocidos y clásicos del pensamiento y la literatura universal. Estas series de folletos se muestran en estrecha relación con otras que comienzan en esas fechas - Bambalinas, Teatro popular, Joyas literarias, Los inmortales, Novela Semanal, La escena, Los intelectuales, Teatro popular y Las pequeñas grandes obras - ; muchas de estas series editadas y vendidas en Boedo 841 por esos años ${ }^{33}$.

Por otro lado, de la mano de la editorial La Protesta y de la nueva editorial Argonauta aparecen los primeros libros anarquistas que superan los cientos de páginas en ediciones bellas y cuidadas. Si bien éstas por lo general se siguen financiando durante largas campañas en búsqueda de suscripciones, al mismo tiempo generan un importante salto en lo que se refiere a ciertas marcas de mejoramiento editorial: con traducciones revisadas, ediciones corregidas y un nuevo interés hacia textos de reflexión teórica de más largo aliento. Como se desprende por las cartas de Luis Juan Guerrero a Max Nettlau y Diego Abad de Santillán, Argonauta nació como una editorial anarquista que a la vez pretendió impulsar ediciones desvinculadas directamente del movimiento bajo el nombre de Minerva, con textos que Santillán y Guerrero consideraban importante poner en circulación $^{34}$. Pero destacamos que — en esta etapa donde comienza a primar el formato libro - se mantienen los objetivos con los que los emprendimientos libertarios insisten con diferenciarse de una empresa comercial: dados por el proyecto propagandístico de concientización e ilustración, la financiación por suscripciones, los precios bajos y la solicitud de hacer circular el texto después de leído ${ }^{35}$. Por su parte, a la par de su influyente

\footnotetext{
${ }^{33}$ Entre los editores y escritores ligados a la Alianza Libertaria Argentina y aquellos alrededor del llamado grupo de Boedo se generaron una serie de colaboraciones aún no suficientemente estudiadas plasmadas en revistas culturales como Dínamo (1924), Extrema Izquierda (1924), La Campana de Palo (1925-1927) e Izquierda (1927-1928), en las cuales por parte del bando anarcófilo parciparon Juan Lazarte, Luis Di Filipo, Elías Castelnuovo, Julio Barcos, Leónidas Barletta, Sebastián Ferrer y José Torralvo, entre otros

${ }^{34}$ Correspondencia disponible en el Fondo de Diego Abad de Santillán del International Institutte of Social History de la cual también existe copia en el Centro de Documentación e Investigación de la Cultura de Izquierda en Buenos Aires.

${ }^{35}$ Resulta ilustrativa al respecto esta carta de Luis Juan Guerrero a Max Nettlau: "Nuestro organismo consiste en una agrupación de afinidad, integrada por un núcleo de camaradas voluntarios en todas las labores concernientes a la difusión de los ideales libertarios. Nuestros propósitos consisten en ir editando continuamente, en castellano, las mejores publicaciones que vayan apareciendo, en los distintos países y en los distintos idiomas, sobre las cuestiones más importantes para nuestros principios. Constituimos pues, no una empresa comercial, sino una organización de propaganda anarquista. Precisamente uno de los móviles que tuvimos al constituirnos en 1920 fue el de evitar que los editores burgueses corriesen con la publicación de obras anarquistas, como ha ocurrido hasta ahora, para mal de todos nosotros, debido al desatino que siempre han mostrado en la publicación de estas obras y al precio excesivo al que siempre han sido vendidas" (2/1/1923) Fondo Nettlau, Arch. Nr. 553 [IISH]. Asimismo pocos años después se presenta la editorial Atlas: "La editorial Atlas, para hacer más extensiva su obra, ha resuelto establecer un amplio servicio de librería, para lo cual ha selecciona de las distintas editoriales del país y del extranjero, las mejores obras científicas y literarias de autores universales. [...] Con el deseo de educar moral y culturalmente al pueblo divulgando conocimientos que eran patrimonios exclusivos de una elite”.
} 
Suplemento (1922-1930), La Protesta, que hasta 1922 sólo había editado unos quince folletos, durante esta década publicará aproximadamente 75 títulos, alcanzando lo que sería su apogeo editorial ${ }^{36}$.

\section{El libro anarquista (1930-1955)}

Como adelantamos, con el correr de las décadas la cantidad y tirada de los periódicos menguaron considerablemente, mientras aumentó el tamaño y la calidad de los libros editados. Excepto frente a algunos últimos hitos que despertarán pequeñas aunque urgentes series de periódicos.

Una vez pasados los años treinta la historiografía libertaria encontrará una serie de últimos hitos locales: las campañas internacionales por Sacco y Vanzetti enlazan aquí con la lucha por los llamados "Presos de Bragado"; la Huelga de la Construcción de 1936 tiene protagonistas anarquistas en el grupo Spartacus; la Revolución y Guerra Civil Española se convierten en la última gran epopeya internacional del anarquismo; en menor medida se recuerda la llamada "Huelga de los locos", la discutida experiencia del anarquismo en los años '70, y, finalmente, el movimiento anarcopunk en Argentina.

Durante la década del treinta, por un lado, el ingreso de autores libertarios a las ediciones populares que había comenzado a través de los folletines se materializaba en las ediciones económicas que realizaba Tor- que en su catálogo ahora incluía a Reclus, Proudhon, De Carlo y "Juan Teófilo" Fichte. En cuanto a los proyectos revisteriles y editoriales libertarios, entre 1931 y 1936, se destaca otro emprendimiento que tiene por partícipe a Abad de Santillán, la colección editorial alrededor de la revista Nervio, que pretendió marcar un giro en cuanto a la producción libertaria anterior. Esta cita, un poco posterior, conserva la autoevaluación que el mismo Santillán expresó en sus obras de 1930 y 1933:

Se han divulgado ideas, no se han pensado; el movimiento argentino fue vehículo excelente, pero no se ha ofrecido al mundo mucho de original. Se ha penetrado en el teatro, en la literatura, en el periodismo y hasta se ha influido en la orientación política durante algunos períodos por el vigor de la agitación callejera, pero no se ha trabajado con un criterio que llamaríamos regional en el terreno del pensamiento. Se ha procedido con más independencia de los hechos... (Abad de Santillán, D., "Bibliografía anarquista argentina", Timón 6, 1938).

\footnotetext{
${ }^{36}$ Desde principios de la década del veinte, los distintos proyectos editoriales libertarios comenzaron a buscar diferenciarse explícitamente de las empresas comerciales. Las críticas por el uso privado de la imprenta no cayeron sobre los editores particulares de esos años - Fueyo y Zuccarelli- u otros proyectos como Argonauta y Atlas, como en cambio sí sucede con un órgano pretendidamente colectivo como La protesta. Sobre el anarquismo argentino de los años veinte, ver los trabajos de Luciana Anapios: "Compañeros, adversarios y enemigos. Conflictos internos en el anarquismo argentino en la década del '20", Entrepasados 32, 2007; "El anarquismo argentino en los años veinte. Tres momentos en el conflicto entre La Protesta y La Antorcha”, Papeles de trabajo 3, 2008.
} 
En su diagnóstico Santillán destaca críticamente el gran volumen de la labor editorial libertaria realizada en Argentina: ella misma no ha generado grandes textos y su producción ha sido en buena medida abstracta y no se ha enfocado en problemas locales y regionales concretos. Su propia respuesta fue la revista y editorial Nervio que editó 18 títulos en seis años. Alrededor de este emprendimiento aparecen ahora jóvenes universitarios, pensadores y literatos, y un cambio en los textos de los propios militantes que intentan enfocarse en mirar variables económicas, sociales y políticas, descentrándose de los "abstractos" textos doctrinarios. Aunque este nuevo enfoque no durará mucho y se impondrá una nueva agenda editorial en referencia a España y un viaje de características épicas para muchos militantes. Mientras, desde 1938, la mayoría de los impulsores de Nervio que no viajaron a España - V. P. Ferrería, A. Llonguet, I. Aguirrebeña, Saúl Kaplan, Costa Iscar y Juan Lazarte - concentraron sus esfuerzos editoriales libertarios en el nuevo sello Imán.

A partir del golpe de Estado del año 1943, el antifascismo argentino anarquista se conjugará en una fuerte oposición al auge del peronismo, que reunía muchas de las inadmisibles características que mostraban los fascismos europeos - líder militar, sindicatos propulsados desde el Estado, censura, educación doctrinaria y otros rasgos autoritarios. Precisamente a partir de 1942, cuando nacen las editoriales Américalee y Tupac, se dará toda una serie de colaboraciones y esfuerzos libertarios para conformar una gran tirada de cuidadas ediciones en tapa dura, con atentas traducciones, prólogos e introducciones, que no dejarán de tener sus lazos con la experiencia de la Unión Democrática y los círculos antifascistas y anticomunistas.

La mencionada editorial Imán -que al menos en sus últimos años imprimía desde los talleres gráficos de Claridad y contaba con grabados de Clement Moreau e introducciones y traducciones de Dardo Cúneo- confluyó en 1941 en el proyecto de Américalee. Esta última se trató de una colección de cultura libertaria fundada por América Scarfó y su compañero Domingo Landolfi, quienes adquirieron la imprenta que había comprado La protesta hacia fines de los años treinta. Aunque, por algunos años principalmente, esta editorial fue impulsada por Diego Abad de Santillán, quien realizó muchas de sus gestiones, introducciones y traducciones. También en 1941, la editorial La Obra - producto del periódico homónimo de Rodolfo González Pacheco- que hasta ese momento solo había publicado unos pocos títulos, suma sus esfuerzos al de los redactores de La Protesta — en aquel momento dos grupos que funcionaban juntos en la Biblioteca José Ingenieros - con el objetivo de lanzar la editorial Tupac.

En esos mismos años, recién fundadas, Américalee y Tupac trazaron enlaces prácticos con el fin de sortear censuras y unificar algunos gastos materiales. Además de las series conjuntas, aparecen libros editados con sellos cruzados como Ediciones La Obra, Tupac y Américalee; y en menor medida algún título como Ediciones Acracia o Losada. Muchas veces estos emprendimientos imprimían el mismo libro desde el taller de Américalee y cada uno le ponía su propio nombre editorial para mantener su sello y llevar a cabo su propia distribución. Como novedad, producto de la ley 11723 de 1938, estas tres 
editoriales, así como las otras empresas del momento, comenzarán a reservarse los derechos de copyright de las obras que publican ${ }^{37}$.

Hasta fines de la década del treinta, la producción local se había disputado el mercado interno con los libros de origen español. De ahora en más, año tras año, la industria nacional del libro aumentará exponencialmente su producción, y, sin duda, Imán, Reconstruir, Tupac y Américalee formaron parte de la llamada "época de oro" del mundo editorial argentino, cuando - desaparecida la industria libresca española debido a la guerra civil - muchas casas editoriales locales ampliaron su alcance a España y otros países de la región. Entre estas editoriales, especialmente Américalee se consolidó con cuantiosas tiradas en gran formato de tapa dura.

Como señala Graciano en su ya citado texto, Américalee combinó autores libertarios con una apertura al público universitario a través de la edición de obras de, por ejemplo, Rodolfo Mondolfo y Benedetto Croce. Incluyó autores socialistas como el nombrado Cúneo y el socialista-libertario italiano Carlo Roselli. Y también editó autores de distintas orientaciones políticas como José Martí, Haya de la Torre, Waldo Frank y Theodore Roosevelt. Si todavía Nervio mantenía un diagnóstico de inminencia revolucionaria e incorporaba cierto bagaje marxista de análisis económico, en cambio, Américalee, en la década siguiente, frente a la segunda guerra, el peronismo y la dictadura soviética, apuntaba a un público antifascista y a la defensa de la democracia, dando lugar en su catálogo a una mayor presencia de nociones liberales y keynesianas.

Mientras Américalee funcionaba en buena medida desligada de instituciones libertarias, en 1946 nace en Rosario la Unión Socialista Libertaria (USL) que realizará una publicación periódica —el Boletín libertario (1948-1976) — y un proyecto editorial en comparación mucho más acotado. Al año siguiente la FACA lanza su revista y sello Reconstruir. Y, como Américalee, Reconstruir, en conjunción con la fuerte oposición al peronismo que llevaba a cabo desde su revista, publicó también autores socialistas y liberales, y en consecuencia sufrió juicios por desacato y persecuciones a sus responsables.

A partir de 1961 y hasta 1976 Reconstruir confluyó en la editorial Proyección con distintas tendencias del anarquismo local que, dejando de lado ciertas diferencias, acordaron emprender una acción conjunta de edición y difusión. Proyección nace en 1961 conformada por Reconstruir, Tupac, y en un principio también por la Federación Anarquista Uruguaya (FAU) y por la Cooperativa Lanera de Sarandí que financiaba buena parte de las tiradas. Proyección conformó un consejo editorial en común que imprimió con copyright desde los talleres de Américalee y alcanzó a lanzar 46 títulos en quince años. Como todas estas editoriales, Proyección retomó la publicación de clásicos anarquistas, a lo que sumó nuevas obras de renovación teórica sobre los temas que se mostraban constantes a los intereses libertarios: sexualidad, cooperativismo y movimiento obrero, entre otros. La

\footnotetext{
${ }^{37}$ A partir de los años ochenta, con la mayor accesibilidad a la posibilidad de hacer copias y la proliferación de funzinez y punkzines, el movimiento editorial libertario se mostrará pionero en discutir desde su práctica el copyright, habitualmente con la frase “Anticopyright: ningún derecho, ningún deber” impreso en la página de guarda de sus ediciones. Recién años después, a principios del dosmil, se establecerá un gran campo de discusión sobre licencias a partir del copyleft y el copyfarleft. Sobre la historia del copyright en Argentina puede verse: Busaniche, Beatriz, Argentina copyleft, Buenos Aires, La Plata, 2010.
} 
editorial funcionó hasta 1976, cuando finalizó sus actividades en el clima de terror impuesto por la dictadura cívico-militar del llamado Proceso de Reorganización Nacional.

\section{Recapitulación}

En resumen, el ciclo editorial anarquista comenzó con la aparición de una serie de novedosos folletos en lo que se refiere tanto a sus modos de circulación y financiación como a sus temas y contenidos. Redacciones de periódicos y bibliotecas obreras se convirtieron en espacios de sociabilidad política por donde se difundieron gran cantidad de impresos locales y muchos otros provenientes del exterior. Desde 1901, la militancia política durante el ciclo de agremiación obrera y las protestas callejeras que se extendieron hasta 1910, en conjunción con la aparición de nuevos publicistas y escritores libertarios, privilegiaron la edición de periódicos gremiales y revistas literarias.

Empíricamente, la cantidad de publicaciones monográficas disminuyó después de esta fecha para revitalizarse hacia 1919 con la llegada de noticias de la Revolución Rusa y el comienzo de un ciclo de fuertes protestas a nivel nacional e internacional. Por lo que entre 1919 y 1923 se registró también un fuerte aumento en la cantidad de folletos editados tanto desde el anarquismo como desde el socialismo y el sindicalismo, cuando, frente a la gran cantidad de discusiones internas, varios grupos las distintas tradiciones de izquierda publicaron más de cincuenta folletos que buscaban redefinir la identidad política de su facción en función de su programa.

Los años posteriores a 1924 fueron momentos en que se dio lugar a los primeros textos historiográficos del anarquismo escrito por sus propios militantes. Y, a la vez, el momento donde aparecen los folletos seriados de cultura libertaria a los que hicimos mención con anterioridad. A partir de las décadas siguientes, el anarquismo editará mucha menos cantidad de periódicos mientras, en contrapartida algunas colecciones de tradición libertarias se insertarán exitosamente en el mercado del libro local e internacional.

Entre 1892 y el 2016 contabilizamos más de ciento cincuenta proyectos editoriales anarquistas. La mayoría fueron efímeros y no pasaron de editar uno o dos títulos y sólo unos pocos superaron la decena. La corta duración de estas iniciativas no debe ser considerada necesariamente como un límite. Muchas veces se trató de grupos o militantes que unían sus esfuerzos para lanzar un texto conjunto frente a una coyuntura o lucha específica. En otros casos las series de libros funcionaron de manera conjunta con una revista o periódico como parte de un proyecto más amplio. Y, casi siempre, los militantes detrás de cada colección realizaban muchas de las tareas propias de las distintas etapas del circuito del libro, ocupándose de la impresión, la edición, la corrección y la distribución.

Las características iniciales del impreso libertario que se mostrarán en buena medida constantes redundaron en lograr libros baratos impulsados con innovadores modos de financiación y distribución y un núcleo de temas en donde radicaba su propuesta amplia de emancipación, y que son los que lo diferenciaron tanto de la prensa comercial como de la cultura impresa socialista. Lejos de presentarse como una propuesta en la cual se genera una empatía simplista con los oprimidos, el intento de la prensa y folletería anarquista ha sido politizar gran parte de las prácticas cotidianas: el trabajo, la escuela, la pareja, el amor, 
la alimentación, la salud ${ }^{38}$. Al priorizar el análisis de la opresión económica como eje explicativo, la caracterización marxista habitual que rige sobre el anarquismo sostuvo que la militancia libertaria solamente ha prosperado durante unos pocos años en las sociedades "atrasadas" no industrializadas. Por el contrario, para matizar esta interpretación, puede pensarse que el éxito del impreso anarquista se debió a la originalidad de su apuesta editorial, claramente visible en su modo de financiación, difusión y en la propuesta de pensar la opresión no sólo dentro de la fábrica sino en muchas de las esferas sociales.

\section{Bibliografía}

\section{Libros, artículos y comunicaciones}

Abad de Santillán, Diego, El anarquismo en el movimiento obrero, Barcelona, Cosmos, 1925.

-----, El movimiento anarquista en la Argentina, desde sus comienzos hasta 1910, Buenos Aires, Argonauta, 1930.

----, La FORA. Ideología y trayectoria del movimiento revolucionario en la Argentina, Buenos Aires, Nervio, 1933.

-----, "Bibliografía anarquista argentina", Timón 6, 1938.

Albornoz, Martín, "Caleidoscopio de palabras. Las reuniones de controversia entre anarquistas y socialistas a finales del siglo XIX y principios del siglo XX”, Sociedad 28, 2009.

Anapios, Luciana, "Una promesa de folletos. El rol de la prensa en el movimiento anarquista en la Argentina (1890-1930)", A Contracorriente 8:2, 2011.

----, "El anarquismo argentino en los años veinte. Tres momentos en el conflicto entre La Protesta y La Antorcha", Papeles de trabajo 3, 2008.

-----, "Prensa y estrategias editoriales del movimiento anarquista en la Argentina de entreguerras", Anuario del Instituto de Historia Argentina, vol. 16, $\mathrm{n}^{\circ}$ 2, 2016.

Barrancos, Dora, "Las 'lecturas comentadas': un dispositivo para la formación de la conciencia contestataria entre 1914-1930", Boletín CEIL XVI, 1987.

Bilsky, Edgardo, La FORA y el movimiento obrero, Buenos Aires, CEAL, 1985.

Buonocuore, Domingo, Libreros, editores e impresores de Buenos Aires. Buenos Aires, Bowker, 1974.

Buonnome, Juan, "Cultura impresa y socialismo. Lecturas sobre la historia de la prensa socialista en tiempos de la Segunda Internacional”, Políticas de la memoria 14, 2012-2013.

Busaniche, Beatriz, Argentina copyleft: La crisis del modelo de derecho de autor y las prácticas para democratizar la cultura, La Plata, UNLP, 2010.

\footnotetext{
${ }^{38}$ En efecto, con anterioridad a la división entre anarquistas y marxistas durante el proceso de la Primera Internacional de Trabajadores, el llamado socialismo romántico aunaba preocupaciones económicas y sociales, sin descuidar estas dimensiones "culturales" de militancia política. Después de esta división, en el lado socialista marxista ha preponderado la lucha contra la opresión económica, mientras en buena medida se consideró al resto como accesorias y secundarias. Toda la historiografía de izquierda, tanto anarquista como socialista, se enfocó en la conformación del movimiento obrero mientras brindaba una importancia secundaria a la presencia de "otras" temáticas consideradas meramente culturales, sin poder valorar entonces gran parte de la apuesta política del anarquismo como movimiento. Ver: Tarcus, H., El socialismo romántico, Buenos Aires, FCE, 2016.
} 
Debray, Regis, "El socialismo y la imprenta: un ciclo vital", New Left Review 46, 2007. de Diego, José Luis, Editores y políticas editoriales en Argentina, 1880-2000, Buenos Aires, FCE, 2006.

de Veyga, Francisco, "Delito Político: El anarquista Planas Virella", Archivos de psiquiatría y criminología V, Buenos Aires, 1906.

De Zagastizábal, Leandro, La edición de libros en la Argentina: una empresa de cultura, Buenos Aires: Eudeba, 1995

Degiovanni, Fernando, Los textos de la patria, Buenos Aires, Beatriz Viterbo, 2007

de la Rosa, Fernanda, "Entre la militancia y los proyectos editoriales: La labor de Diego Abad de Santillán dentro del anarquismo argentino, 1920-1930". IV Jornadas de Historia de las Izquierdas, Buenos Aires, CeDInCI, 2007.

Di Stefano, Mariana, El lector libertario, Buenos Aires, Eudeba, 2013.

Doeswijk, Andreas, Los anarcobolcheviques rioplantenses, Buenos Aires, CeDInCI, 2013.

Domínguez Rubio, Lucas, "La edición de libros y folletos en la conformación del anarquismo argentino", Actas del Primer Coloquio Argentino de Estudios sobre el Libro y la Edición, http://coloquiolibroyedicion.fahce.unlp.edu.ar/actas/Dominguez/view

Ferrer, Christian - Albornoz, Martín (Selección y Prólogo), Folletos anarquistas en Buenos Aires, Buenos Aires: Biblioteca Nacional, 2015.

Fernández Cordero, Laura, "Un ejercicio de lectura sobre el concierto de la prensa anarquista a partir de Mijail Bajtin (Argentina, 1895-1925)", Adversus X, 2013.

Gilimón, Eduardo, Hechos y comentarios, Montevideo, [s.n.], 1911.

Graciano, Osvaldo, "La escritura de la realidad. Un análisis de la tarea editorial y del trabajo intelectual del Anarquismo argentino, entre los años 30 y el Peronismo", Izquierdas 12, 2012.

Gutiérrez, Leando y Romero, José Luís, "Buenos Aires en la entreguerra: libros baratos y cultura de los sectores populares", en Diego Armus (comp.), Mundo urbano y cultura popular: Estudios de historia social, Buenos Aires, Sudamericana, 1990.

Ingenieros, José, "La Internacional en Sud América: datos que servirán para la historia del socialismo", Almanaque socialista de La Vanguardia, 1899

Merbilháa, Margarita, "La época de organización del espacio editorial". José Luis de Diego (dir.). Editores y políticas editoriales en Argentina, 1880-2000, Buenos Aires, FCE, 2006.

Nettlau, Max, "Contribución a la bibliografía anarquista de la América Latina hasta 1914", Certamen Internacional de La Protesta, Buenos Aires, La Protesta, 1927.

Oved, Iacov, El anarquismo y el movimiento obrero en Argentina, México, Siglo XXI, 1978.

Pastormerlo, Sergio, "El surgimiento de un mercado editorial", en De Diego, J. L. (dir.), Editores y políticas editoriales en Argentina: 1880-2000, Buenos Aires: FCE, 2006.

Pérez, Pablo; Villasenin, Hernán; Jofre, Liliana, "Las armas y las letras. Un recorrido por las ediciones anarquistas", La Biblioteca 2, 2006.

Pittaluga, Roberto, Soviets en Buenos Aires, Buenos Aires, Prometeo, 2015.

Prieto, Adolfo, El discurso criollista en la formación de la Argentina moderna, Buenos Aires, Sudamericana, 1988.

Romero, José Luís, "Sociedades barriales, bibliotecas populares y cultura de los sectores populares: Buenos Aires, 1920-1945”, Desarrollo económico 113, 1989.

Sarmiento, N., Historia del Libro y de las Bibliotecas Argentinas. Buenos Aires: Imp. Luis Veggia, 1930

Sik, Eugenia, "La creación de bibliotecas durante el apogeo del anarquismo argentino (1898-1905)" [mimeo] [en prensa].

Suriano, Juan, Anarquistas, Buenos Aires, Manantial, 2001.

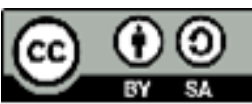


Tarcus, Horacio, Marx en la Argentina, Buenos Aires, Siglo XXI, 2007.

-----, Diccionario biográfico de la izquierda argentina, Buenos Aires, Emecé, 2007.

-----, "La Biblioteca Popular de Bartolomé Victory y Suárez, primera editorial de la izquierda argentina. 1864-65", Actas del primer coloquio sobre el libro y la edición 2012, La Plata: UNLP, 2013.

-----, El socialismo romántico, Buenos Aires, FCE, 2016.

Tripaldi, Nicolás, "Origen e inserción de las bibliotecas obreras en el entorno bibliotecario argentino", Libraria 1, Buenos Aires, 1997.

Valadés, José, "Documentos para la historia del anarquismo en América", Certamen Internacional de La Protesta, Buenos Aires, La Protesta, 1927.

Zaragoza Ruvira, Gonzalo, Anarquismo argentino (1876-1902), Madrid, Ediciones de la Torre, 1996. 